\title{
Allogeneic Serum Eye Drops for the Treatment of Dry Eye Patients with Chronic Graft-Versus-Host Disease
}

\author{
Kyung-Sun $\mathrm{Na}^{1,2}$ and Man Soo Kim²
}

\begin{abstract}
Purpose: To evaluate the therapeutic effect of allogeneic serum eye drops for the treatment of dry eye in patients with chronic graft-versus-host disease (cGVHD) following bone marrow transplantation.

Methods: Sixteen patients with cGVHD following allogeneic hematological stem cell transplantation (allo-SCT) and diagnosed with dry eye syndrome refractory to conventional treatment were prospectively enrolled in this study. Allogeneic serum eye drops were obtained from healthy related donors after serologic testing. Symptom scores, tear breakup time (tBUT), the Schirmer test without anesthesia (Schirmer I test), tear osmolarity, corneal staining score, impression cytology grade, and goblet cell densities were evaluated before and 4 weeks after administration of allogeneic serum eye drops.

Results: Enrolled patients included 6 females and 10 males between 20 and 61 years of age (mean age, $37.2 \pm 11.6$ years). After 4 weeks of treatment, the Ocular Surface Disease Index (OSDI) symptom scores decreased significantly (32.5-8.9). Tear osmolarity showed a significant decrease from 311.1 to 285.1 milliosmol. The corneal staining scores significantly decreased from 2.5 to 1.8. Impression cytology grade and goblet cell density improved from 3.4 to 2.4 and from 90.6 to $122.6 \mathrm{cell} / \mathrm{mm}^{2}$, respectively. tBUT also significantly improved from 2.9 to $4.4 \mathrm{~s}$, and the Schirmer I test results showed improvement, but lacked statistical significance (1.7-2.4 mm). No significant side effects were detected as a result of the allogeneic serum treatment during the follow-up period. Conclusions: Allogeneic serum can be used for the treatment of severe dry eye in patients with cGVHD when autologous serum is unavailable. Care should be taken to avoid the risk of blood-borne diseases.
\end{abstract}

\section{Introduction}

A LLOGENEIC HEMATOLOGiCAL STEM cell transplantation (allo-SCT) is used as a curative therapy for a large number of malignant and nonmalignant hematological diseases. Graft-versus-host disease (GVHD), which can be acute or chronic, is the major cause of morbidity and mortality following allo-SCT. Approximately $40 \%$ of human leukocyte antigen (HLA)-matched patients develop acute systemic GVHD, and $30 \%-70 \%$ of patients develop chronic systemic GVHD. Chronic GVHD (cGVHD) develops 100 days after allo-SCT, and the sites most commonly affected are the skin, mouth, liver, gastrointestinal tract, lung, and eye. ${ }^{1-3}$

Ocular GVHD develops in $40 \%-60 \%$ of patients after alloSCT and can cause severe ocular surface diseases, including dry eye. ${ }^{4}$ Keratoconjunctivitis sicca (KCS), or dry eye associated with CGVHD, is one of the major complications after allo-SCT; KCS has a significant impact on a patient's quality of life and can lead to blindness. Dry eye with cGVHD usually results in conjunctival inflammation and chronic blepharitis, which causes punctuate keratopathy, epithelial erosion, and even corneal perforation. KCS can also cause conjunctival fibrosis, lid abnormality, atrophy of meibomian glands, loss of eyelashes, and stenosis of the lacrimal punctum. ${ }^{5-7}$ Therapeutic options include artificial tears, therapeutic contact lenses, punctual plugs, topical or systemic corticosteroids, and other immunosuppressive drugs. ${ }^{3,6}$

Previous reports have demonstrated that autologous serum eye drops are effective for treating dry eye disease and have also been used for severe ocular disorders, including Sjogren and non-Sjogren syndrome, ocular surface disorders such as cicatricial pemphigoid syndrome, Steven-Johnson syndrome, and superior limbic keratitis. ${ }^{8-12}$ These eye drops contain various substances, such as epidermal growth factor, vitamin A, transforming growth factor- $\beta$, and fibronectin. ${ }^{10,13-15}$ The efficacy and safety of serum eye drops for dry eye disease in patients with cGVHD have been previously investigated. ${ }^{14,16-18}$ However, autologous serum may be unavailable for patients with cGVHD, because Asians commonly believe that frequent venipuncture results in general

Departments of ${ }^{1}$ Health Promotion Center and ${ }^{2}$ Ophthalmology and Visual Science, College of Medicine, The Catholic University of Korea, Seoul, St. Mary's Hospital, Seoul, Korea.

The English in this document has been checked by two experienced editors, both native speakers of English (www.editage.co.kr). 
weakness or increases the risk of bacterial infection. For these patients, practitioners suggest the use of allogeneic serum eye drops from related donors as an alternative treatment. A previous case report on the allogeneic serum use for the treatment of severe dry eye in patients with CGVHD concluded that allogeneic serum is a good alternative treatment. ${ }^{14}$ To confirm these encouraging results, we prospectively evaluated the safety and efficacy of allogeneic serum drops for moderate-to-severe dry eye in patients with cGVHD.

\section{Methods}

Sixteen individuals with cGVHD after allo-SCT and who had been diagnosed with dry eye syndrome refractory to conventional treatment were included in this study. The Institutional Review Board/Ethics committee approval was obtained, and the study protocol followed the guidelines of the Declaration of Helsinki. Informed consent was obtained from the patients and the related donors. The inclusion criteria were as follows: the Schirmer test without anesthesia (Schirmer I test) result of less than $5 \mathrm{~mm} / 5 \mathrm{~min}$; low tear breakup time (tBUT) of $<5 \mathrm{~s}$; moderate-to-severe superficial punctate keratitis, defined as a corneal staining score of more than 2; and symptoms of ocular irritation assessed by an Ocular Surface Disease Index (OSDI) score of more than 30 (scale of 0-59). Exclusion criteria included active ocular infection or inflammation not associated with dry eye, drug toxicity, or eyelid abnormalities. The following common examinations for dry eye disease were performed by 1 investigator (K.S. Na) at every visit: symptom scores, tBUT, the Schirmer test without anesthesia, cornea staining, conjunctival impression cytology, and tear osmolarity.

Subjective symptoms were graded on a numerical scale from 0 through 4 using the OSDI score: 0 , none of the time; 1 , some of the time; 2 , half of the time; 3 , most of the time; 4 , all of the time. The tBUT was measured after placing a sodium fluorescein paper at the lower tarsal conjunctiva. The patients were asked to blink, and the time before the defect appeared in the stained tear film was measured and recorded as the tBUT. A 5-min Schirmer test was performed using sterile strips (ColorBar ${ }^{\mathrm{TM}}$ Schirmer tear test; EagleVision ${ }^{\circledR}$ ) without anesthesia. The Schirmer strip was placed at the notch of the inferior fornix, and after $5 \mathrm{~min}$, the strip was removed and measured at the point of maximum wetting.

For corneal fluorescein staining, the entire cornea was examined by slit-lamp evaluation with a yellow barrier filter and cobalt blue illumination. Anterior segment photographs were taken, and stains were graded using the Oxford Scheme 6-point scale (from 0 through 5).

Impression cytology was performed at the lower temporal bulbar conjunctiva using a cellulose acetate filter paper (MFS membrane filters; Advantec MFS). Papers were fixed in absolute alcohol, stained with periodic acid-Schiff and hematoxylin stain, and photographed at a magnification of $400 \times$. Squamous metaplasia was graded from 0 through 6 using the Tseng classification. ${ }^{19}$ Tear osmolarity was measured using a laboratory-on-a-chip device, which simultaneously collected and analyzed the electrical impedance of a 50-nL tear sample from the inferior lateral meniscus (TearLab Osmolarity System).

Allogeneic serum was taken from a patient's spouse, parent, or offspring (over 20 years old). The serum was screened using standard tests used in blood bank screening to check for blood-borne diseases such as human immunodeficiency virus, hepatitis B virus, and hepatitis C virus. Allogeneic serum was prepared using the following previously described method: venesection was performed at the antecubital fossa under aseptic conditions, and $20 \mathrm{~mL}$ of whole blood was collected in sterile containers. ${ }^{10}$ The blood was centrifuged immediately at $1500 \mathrm{rpm}$ for $10 \mathrm{~min}$ to obtain serum. Serum was diluted with preservative artificial tears (Refresh Plus ${ }^{\circledR}$, ALLERGAN) to obtain a final concentration of $20 \%$. A measure of $20 \mu \mathrm{L}$ of $0.3 \%$ ofloxacin was added to the diluted serum. The entire solution was divided into sterile $10-\mathrm{mL}$ bottles and stored in a freezer at $-20^{\circ} \mathrm{C}$. Once thawed, the serum eye drops were stored in a refrigerator at $4^{\circ} \mathrm{C}$ and were used within 1 week.

Patients used allogeneic serum eye drops 6-8 times daily. Nonpreservative artificial tears were used as an adjunct treatment. At the 4-week follow-up, a slit-lamp examination to evaluate the corneal and conjunctival change, the Schirmer test without anesthesia, and conjunctival impression cytology were performed; tBUT and tear osmolarity were also evaluated.

Categorical variable changes from baseline were evaluated using the Wilcoxon signed-rank test, and changes in continuous variables were analyzed using a paired $t$ test. A two-sided test with a $P$ value of $<0.05$ was considered statistically significant.

\section{Results}

When the prospective treatment began, only 1 patient received systemic cyclosporine A and steroid in combination, and 10 received systemic steroid treatment alone. Patient characteristics are shown in Table 1. Six females and 10 males were included in this study, with ages ranging between 20 and 61 years (mean, 37.2 \pm 11.6 years). Pretreatment diagnosis of the enrolled patients was acute myelogenous leukemia in 10 patients, acute lymphocytic leukemia in 3 patients, myelodysplastic syndrome in 1 patient, chronic lymphocytic leukemia in 1 patient, and chronic myelogenous leukemia in 1 patient. The duration from the allo-SCT to dry eye diagnosis ranged from 4 to 25 months (mean, $9.8 \pm 5.5$ months). The follow-up period ranged from 3 to 24 months (mean, $11.6 \pm 7.0$ months).

Changes in symptoms and signs after administration of allogeneic serum eye drops are shown in Table 2. After 4 weeks of treatment, the OSDI symptom scores decreased significantly (from 32.5 to 8.9 ), and the most notable changes occurred in photophobia and blurred vision. Tear osmolarity showed a significant decrease from 311.1 to 285.1 mOSM; corneal staining score significantly decreased from 2.5 to 1.8; and impression cytology grade and goblet cell density improved from 3.4 to 2.4 and 90.6 to $122.6 \mathrm{cell} / \mathrm{mm}^{2}$, respectively (Fig. 1). tBUT also significantly improved from 2.9 to $4.4 \mathrm{~s}$, and the Schirmer I test results showed improvement, but did not reach statistical significance (from 1.7 to $2.4 \mathrm{~mm}$ ). During the follow-up period, no significant side effects were detected as a result of the allogeneic serum treatment.

\section{Discussion}

Dry eye is the most frequent ocular complication of cGVHD, and the median time from allo-SCT to a dry eye diagnosis is 171 days. Administration of immunosuppressant 
Table 1. Characteristics of Patients with Chronic Ocular Graft-Versus-Host Disease

\begin{tabular}{|c|c|c|c|c|c|c|c|}
\hline No. & Age & Sex & Diagnosis & $\begin{array}{l}\text { Time from } \\
\text { SCT to dry eye }\end{array}$ & $\begin{array}{l}\text { cGVHD other } \\
\text { than eyes }\end{array}$ & $\begin{array}{c}\text { Systemic } \\
\text { immunosuppressant }\end{array}$ & $\begin{array}{l}\text { Follow-up after } \\
\text { allogeneic serum }\end{array}$ \\
\hline 1 & 31 & $\mathrm{~F}$ & AML & 4 & Oral, Lung & Steroid & 24 \\
\hline 2 & 51 & $\mathrm{M}$ & CLL & 12 & Oral, GI & Steroid & 12 \\
\hline 3 & 46 & $\mathrm{M}$ & ALL & 6 & & & 6 \\
\hline 4 & 61 & $\mathrm{~F}$ & ALL & 12 & Oral & & 6 \\
\hline 5 & 37 & M & MDS & 5 & & & 5 \\
\hline 6 & 30 & $\mathrm{M}$ & ALL & 11 & Oral & Steroid & 4 \\
\hline 7 & 45 & $\mathrm{M}$ & CML & 16 & Lung, GI & Steroid & 9 \\
\hline 8 & 41 & $\mathrm{M}$ & AML & 25 & GI & Steroid & 6 \\
\hline 9 & 27 & $\mathrm{M}$ & AML & 13 & Oral & & 8 \\
\hline 10 & 47 & $\mathrm{M}$ & AML & 12 & Oral & Steroid & 12 \\
\hline 11 & 45 & $\mathrm{~F}$ & AML & 6 & Oral & Steroid & 24 \\
\hline 12 & 37 & $\mathrm{~F}$ & AML & 8 & Oral, Skin, GI & Steroid, CsA & 18 \\
\hline 13 & 25 & $\mathrm{M}$ & AML & 4 & Oral, Skin & Steroid & 17 \\
\hline 14 & 21 & $\mathrm{M}$ & AML & 5 & Skin & & 3 \\
\hline 15 & 31 & $\mathrm{~F}$ & AML & 7 & & & 11 \\
\hline 16 & 20 & $\mathrm{~F}$ & AML & 10 & Oral, GI & Steroid & 20 \\
\hline
\end{tabular}

SCT, stem cell transplantation; cGVHD, chronic graft-versus-host disease; AML, acute myelogenous leukemia; ALL, acute lymphocytic leukemia; CML, chronic myeloid leukemia; CLL, chronic lymphocytic leukemia; MDS, myelodysplastic syndrome; CsA, cyclosporine A.

drugs for GVHD prophylaxis is usually completed at approximately 180 days after SCT, and cGVHD frequently develops during tapering or discontinuation of the immunosuppressive agent. ${ }^{20,21}$ CGVHD is a multiorgan inflammatory disorder that resembles an autoimmune disease. These inflammatory diseases may cause fibrosis, stenosis, and atrophy of the involved tissue, including the eye, liver, lung, skin, and gastrointestinal tract. Chronic ocular GVHD causes inflammatory destruction of the conjunctiva and the lacrimal gland with fibrosis, resulting in tear deficiency and, often, damaged surfaces. ${ }^{22-24}$ This condition can also cause meibomian gland dysfunction with subsequent atrophy, contributing to a poor quality of the tear film. ${ }^{25}$ Treatment options for ocular GVHD are anti-inflammatory agents, such as topical corticosteroids, cyclosporine A, autologous serum eye drops, tacrolimus, and tranilast; symptomatic treatments include artificial tears, punctal occlusion, or therapeutic contact lenses. Topical corticosteroid use results in improvement of the conjunctival signs and, to some extent, corneal staining. However, patients should be monitored for adverse effects, including corneal epithelial defect, stromal thinning, cataract, secondary infection, and glaucoma. ${ }^{26}$ Topical cyclosporine $\mathrm{A}$ is also used for resolving or relieving
cGVHD symptoms, and large-scale studies evaluating this type of treatment are being conducted. ${ }^{27-29}$ Clinical data are currently insufficient for evaluating the efficacy and safety of topical tacrolimus and tranilast, and these drugs are generally not easy to use for ophthalmologists. ${ }^{30,31}$

Growth factors, vitamins, and neuropeptides secreted from lacrimal glands support the proliferation, migration, and differentiation of the ocular surface epithelia. As part of inflammatory processes, additional proteins, including the adhesion factor fibronectin, complement factors, and antimicrobial proteins are secreted from conjunctival vessels. Serum and other body fluids also contain similar components as those present in tear substitutes, which contain various growth factors, vitamins, and immunoglobulins, some in higher concentrations than those present in natural tears. Therefore, autologous serum eye drops can supply important factors for maintaining corneal and conjunctival integrity, which artificial tears fail to provide. The use of these drops was first reported in 1984, ${ }^{13}$ and they have been used for treating severe dry eye disease, persistent epithelial defect, and for support during ocular surface reconstruction. Successful outcomes have been achieved after use of serum eye drops for severe dry eye disease associated with cGVHD.

Table 2. Changes of Symptoms and Signs After Allogeneic Serum Eye Drops in Patients with Chronic Ocular Graft-Versus-Host Disease

\begin{tabular}{lcrc}
\hline & & Four weeks after allogeneic \\
serum eye drops & Before treatment & $20.6 \pm 5.6$ & $P_{\text {-value }}$ \\
\hline OSDI score & $32.5 \pm 8.9$ & $4.4 \pm 2.2$ & $<0.005$ \\
tBUT(s) & $2.9 \pm 1.9$ & $2.4 \pm 1.9$ & 0.04 \\
Schirmer I test (mm) & $1.7 \pm 2.3$ & $285.1 \pm 5.8$ & 0.32 \\
Tosm (mOSM) & $311.1 \pm 15.9$ & $1.8 \pm 0.7$ & $<0.005$ \\
Corneal staining score & $2.5 \pm 0.6$ & $2.4 \pm 0.6$ & $<0.005$ \\
IC grade & $3.4 \pm 0.5$ & $122.6 \pm 49.2$ & $<0.005$ \\
Goblet cell density (cells $/ \mathrm{mm}^{2}$ ) & $90.6 \pm 44.1$ & & $<0.005$ \\
\hline
\end{tabular}

astatistics: paired $t$-test.

OSDI score, Ocular Surface Disease Index score; tBUT, tear breakup time; Tosm, tear osmolarity; IC, impression cytology; mOSM, milliosmol. 

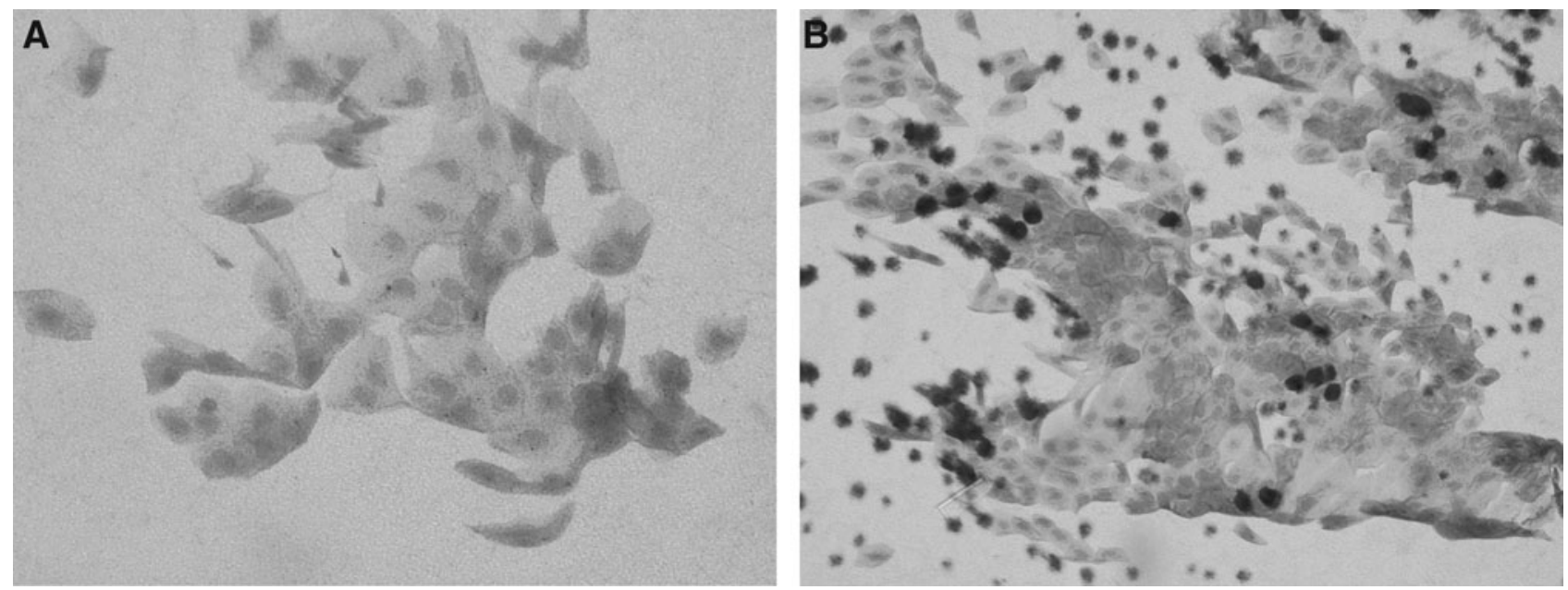

FIG. 1. Representative conjunctival impression cytology specimens before and after 4 weeks of allogeneic serum eye drop use. (A) Before treatment. High-grade squamous metaplasia with the presence of a few goblet cells. (B) Four weeks after allogeneic serum eye drop use. Note obvious improvement of squamous metaplasia and increase in the number of goblet cells.

Ogawa et al. investigated the efficacy and safety of autologous serum eye drops after treating 14 patients with severe dry eye associated with cGVHD. ${ }^{17}$ A total of 14 patients with severe dry eye associated with cGVHD were enrolled, and the results showed that a significant improvement was observed in complaints scores, corneal staining score, and tear dynamics. Kojima et al. conducted a randomized in vivo study and an in vitro study and concluded that albumin, a major protein in serum, ameliorated ocular surface damage. ${ }^{15}$ Rocha et al. reported a beneficial clinical effect of autologous serum application in 2 cases of dry eye with GVHD. ${ }^{16}$ Yoon et al. also reported that in 12 patients with ocular GVHD, use of umbilical cord serum eye drops resulted in a therapeutic effect. ${ }^{18}$ However, autologous serum is not available when patients have active systemic inflammation, fear of venipuncture, and if they are infants or elderly. Frequently, patients with ocular GVHD have cGVHD in locations other than the eyes, and in those cases, poor systemic conditions such as malnutrition or dyspnea can cause patients to hesitate in using autologous serum for the treatment of dry eye disease. In those cases, an alternative treatment method, such as allogeneic serum eye drops from healthy family members, is recommended for ocular surface restoration. Chiang et al. reported 2 cases of successful allogeneic serum eye drop treatment for severe dry eye with cGVHD. ${ }^{14}$ In our prospective study, both subjective and objective symptoms and signs showed marked improvement in 4 weeks, which is similar to the results of autologous serum or umbilical serum eye drops. The amount of aqueous tear was not significantly improved because once the lacrimal gland fibrosis has occurred, it is impossible to restore it. However, the goblet cell increase may lead to the improvement of ocular surface condition and dry eye symptoms.

In this study, only family members with no history of blood-transmitted diseases or active inflammation were included, and serologic test results were checked before venipuncture. It is especially important to test for bloodtransmitted diseases such as HIV and hepatitis B and C before using allogeneic serum. Blood type was not considered because $\mathrm{ABO}$ type was considered to have no effect in healing corneal or conjunctival epithelium. Few previous studies have reported serious complications after serum eye drop use. Fox et al. reported a possibility of sclera vasculitis or scleral melting in patients with rheumatoid arthritis. ${ }^{13}$ Circulating antibodies from serum eye drops and resident corneal antibodies can theoretically form immune complexes; a small case series of temporary bacterial conjunctivitis, which was resolved with proper antibiotic treatment, has been reported. ${ }^{16,17,32}$ However, these complications can occur during the natural course of the disease in patients with rheumatoid arthritis, tear deficiency, and soft-bandage contact lenses. In conclusion, allogeneic serum can be used for treating severe dry eye in patients with cGVHD when autologous serum is unavailable. Care should be taken to avoid the risk of blood-borne diseases, and ophthalmologists and patients should be aware of signs of ocular infection.

\section{Acknowledgments}

The first author (K.S. Na) was supported by the National Research Foundation of Korea (2011-0027157), Seoul, Korea, for this research.

\section{Author Disclosure Statement}

Authors have no commercial interest in the subject of the article or in entities discussed in the article. No competing financial interests exist.

\section{References}

1. Balaram, M., Rashid, S., and Dana, R. Chronic ocular surface disease after allogeneic bone marrow transplantation. Ocul. Surf. 3:203-211, 2005.

2. Ferrara, J.L., et al. Graft-versus-host disease. Lancet. 373: 1550-1561, 2009.

3. Riemens, A., et al. Current insights into ocular graft-versushost disease. Curr. Opin. Ophthalmol. 21:485-494, 2010.

4. Westeneng, A.C., et al. Ocular graft-versus-host disease after allogeneic stem cell transplantation. Cornea. 29:758-763, 2010. 
5. Saito, T., et al. Ocular manifestation of acute graft-versushost disease after allogeneic peripheral blood stem cell transplantation. Int. J. Hematol. 75:332-334, 2002.

6. Ogawa, Y., and Kuwana, M. Dry eye as a major complication associated with chronic graft-versus-host disease after hematopoietic stem cell transplantation. Cornea. 22:S19-S27.

7. Khanal, S., and Tomlinson, A. Tear physiology in dry eye associated with chronic GVHD. Bone Marrow Transplant. 47:115-119, 2012.

8. Dogru, M., and Tsubota, K. Pharmacotherapy of dry eye. Expert Opin. Pharmacother. 12:325-334, 2011.

9. Geerling, G., et al. Autologous serum and alternative blood products for the treatment of ocular surface disorders. Ophthalmologe. 105:623-631, 2008.

10. Geerling, G., Maclennan, S., and Hartwig, D. Autologous serum eye drops for ocular surface disorders. Br. J. Ophthalmol. 88:1467-1474, 2004.

11. O'Brien, P.D., and Collum, L.M. Dry eye: diagnosis and current treatment strategies. Curr Allergy Asthma Rep. 4:314319, 2004.

12. Samarkos, M., and Moutsopoulos, H.M. Recent advances in the management of ocular complications of Sjögren's syndrome. Curr. Allergy Asthma Rep. 5:327-332, 2005.

13. Fox, R.I., et al. Beneficial effect of artificial tears made with autologous serum in patients with keratoconjunctivitis sicca. Arthritis Rheum. 27:459-461, 1984.

14. Chiang, C.C., et al. Allogeneic serum eye drops for the treatment of severe dry eye in patients with chronic graftversus-host disease. Cornea. 26:861-863, 2007.

15. Kojima, T., et al. Autologous serum eye drops for the treatment of dry eye diseases. Cornea. 27 Suppl 1:S25-S30, 2008.

16. Rocha, E.M., et al. GVHD dry eyes treated with autologous serum tears. Bone Marrow Transplant. 25:1101-1103, 2000.

17. Ogawa, Y., et al. Autologous serum eye drops for the treatment of severe dry eye in patients with chronic graft-versushost disease. Bone Marrow Transplant. 31:579-583, 2003.

18. Yoon, K.C., et al. Therapeutic effect of umbilical cord serum eye drops for the treatment of dry eye associated with graftversus-host disease. Bone Marrow Transplant. 39:231-235, 2007.

19. Tseng, S.C. Staging of conjunctival squamous metaplasia by impression cytology. Ophthalmology. 92:728-733, 1985.

20. Vigorito, A.C., et al. National Institutes of Health. Evaluation of NIH consensus criteria for classification of late acute and chronic GVHD. Blood. 114:702-708, 2009.

21. Shulman, H.M., et al. Chronic graft-versus-host syndrome in man. A long-term clinicopathologic study of 20 Seattle patients. Am. J. Med. 69:204-217, 1980.
22. Jack, M.K., and Hicks, J.D. Ocular complications in highdose chemoradiotherapy and marrow transplantation. Ann. Ophthalmol. 13:709-711, 1981.

23. Russo, P.A., Bouchard, C.S., and Galasso, J.M. Extendedwear silicone hydrogel soft contact lenses in the management of moderate to severe dry eye signs and symptoms secondary to graft-versus-host disease. Eye Contact Lens. 33:144-147, 2007.

24. Hassan, A.S., et al. Lacrimal gland involvement in graftversus-host disease: a murine model. Invest. Ophthalmol. Vis. Sci. 46:2692-2697, 2005.

25. Ogawa, Y., et al. A significant role of stromal fibroblasts in rapidly progressive dry eye in patients with chronic GVHD. Invest. Ophthalmol. Vis. Sci. 42:111-119, 2001.

26. Robinson, M.R., et al. Topical corticosteroid therapy for cicatricial conjunctivitis associated with chronic graft-versushost disease. Bone Marrow Transplant. 33:1031-1035, 2004.

27. Wang, Y., et al. Ocular surface and tear functions after topical cyclosporine treatment in dry eye patients with chronic graft-versus-host disease. Bone Marrow Transplant. 41:293-302, 2008.

28. Kurt, R.A., et al. Topical cyclosporine-A in dry eye associated with chronic graft versus host disease. Ann. Ophthalmol. (Skokie). 41:166-169, 2009.

29. Lelli, G.J., Jr., Musch, D.C., et al. Ophthalmic cyclosporine use in ocular GVHD. Cornea. 25:635-638, 2006.

30. Tam, P.M., et al. Topical $0.03 \%$ tacrolimus ointment in the management of ocular surface inflammation in chronic GVHD. Bone Marrow Transplant. 45:957-958, 2010.

31. Ogawa, Y., et al. Topical tranilast for treatment of the early stage of mild dry eye associated with chronic GVHD. Bone Marrow Transplant. 45:565-569, 2010.

32. Tananuvat, N., et al. Controlled study of the use of autologous serum in dry eye patients. Cornea. 20:802-806, 2001.

Received: January 3, 2012

Accepted: May 12, 2012

Address correspondence to: Dr. Man Soo Kim Department of Ophthalmology and Visual Science College of Medicine

The Catholic University of Korea, Seoul St. Mary's Hospital

\#505 Ban-Po-Dong, Seo-Cho-Gu Seoul 137-040

Korea

E-mail: mskim@catholic.ac.kr 